\title{
La regresión de la hipertrofia ventricular izquierda con antihipertensivos reduciría el riesgo de muerte súbita
}

Regression of Electrocardiographic Left Ventricular Hypertrophy During Antihypertensive Therapy and Reduction in Sudden Cardiac Death: the LIFE Study

\section{Objetivo}

Evaluar si la regresión de la hipertrofia de ventrículo izquierdo (HVI) reduce el riesgo de muerte súbita (MS).

\section{Diseño}

Subanálisis del ensayo clínico aleatorizado y doble ciego LIFE, que comparó losartán con atenolol'

\section{Lugar}

Gothenburgo, Suecia.

\section{Pacientes}

Pacientes de 55 a 80 años de edad con hipertensión arterial (HTA) e HVI tratados con losartán o atenolol seguidos durante un promedio de 4,8 años.

\section{Evaluación de factores pronósticos}

La MS fue definida como aquella muerte de aparición brusca e inesperada y no debida a infarto agudo de miocardio (IAM) insuficiencia cardiaca u otra causa identificable. La HVI se diagnosticó por los criterios electrocardiográficos ${ }^{2}$ del "producto de Cornell" (PC) tomando como umbral 2440mm.ms y/o de Sokolow-Lyon (SL) con un umbral $38 \mathrm{~mm}$. Se ajustó por factores de riesgo, tratamiento asignado, frecuencia cardiaca, tasa albúmina/creatinina, insuficiencia cardiaca, fibrilación auricular y ocurrencia de infarto de miocardio.
Wachtell K, et al. Circulation 2007:116(7):700-5.

Resultados principales

Presentaron MS el 2,1\% de los pacientes. Luego del ajuste, el tiempo de ausencia de HVI bajo tratamiento se asoció con una disminución de riesgo de MS: por cada descenso de un desvío estándar (DE) del PC (1050 mm.ms) se redujo un $19 \%$, por cada $10,5 \mathrm{~mm}$ de descenso del de SL un $18 \%$ y por el descenso de ambos, un $30 \%$. Ver tabla 1.

Tabla 1: riesgo relativo de muerte súbita por cada desvío estándar de descenso de los valores electrocardiográficos de hipertrofia ventricular izquierda.

\begin{tabular}{l|c|c|c|}
\hline & $\begin{array}{c}\text { Produclo de } \\
\text { Coornell }\end{array}$ & Sokolow-Lyon & Ambos \\
\hline $\begin{array}{l}\text { Riesgo (HR) de Muerte } \\
\text { Súbita (IC95\%) }\end{array}$ & $0,81(0,73-0,90)$ & $0,82(0,70-0,98)$ & $0,70(0,54-0,92)$ \\
\hline
\end{tabular}

\section{Conclusión}

El tiempo transcurrido sin HVI electrocardiográfica bajo tratamiento antihipertensivo se asoció a una reducción del riesgo de MS, independientemente del tratamiento, de la reducción de la TA, de la presencia de enfermedad coronaria y otros factores de riesgo en pacientes hipertensos con HVI.

Palabras Claves: hipertrofia, muerte súbita, electrocardiografía Key Words: left ventricular hypertrophy, sudden death, electrocardiography Fuente de financiamiento: Merck \& $\mathrm{Co}$, Inc.

\section{Comentario}

La regresión de la HVI se asocia independientemente con una reducción de eventos combinados como mortalidad cardiovascular, IAM y accidente cerebrovascular ${ }^{3}$. Los mecanismos biológicos posibles se vincularían con un menor consumo de oxígeno y de ese modo, un umbral más alto para el desarrollo de arritmias letales.

Este estudio tiene la fortaleza de haber sido aleatorizado y de haber ajustado por covariables. Su limitación es que contó con una población muy seleccionada de alto riesgo por sus características basales y por el diagnóstico electrocardiográfico de $\mathrm{HVI}$, de sensibilidad y especificidad limitada (su capacidad diagnóstica es aceptable solamente ante hipertrofia severa y no permite cuantificar su grado ${ }^{4}$ ). Nuestros pacientes suelen tener HTA e HVI con diferente severidad, por lo que necesitamos herramientas que nos permitan hacer un diagnóstico más precoz para estratificarlos mejor, por ejemplo la ecocardiografía, que ha demostrado correlacionar bien la masa ventricular izquierda y la anatomía patológica ${ }^{5,6}$.

\section{Conclusiones del comentador}

Sería necesario conocer si la regresión de la HVI se asocia con una reducción de la MS en todo el espectro de pacientes con HVI. En esta línea hay estudios que han demostrado reducción de eventos, pero no MS, probablemente por el menor riesgo basal'. Igualmente, la HVI es considerada como un "daño de órgano blanco" en hipertensos, lo que nos obliga a intensificar el tratamiento y a alcanzar metas más exigentes de control de la $T A^{8}$.

Anbíal Arias [ Servicio de Cardiología. Hospital Italiano de Buenos Aires. anibal.arias@hospitalitaliano.org.ar ]

Ver glosario*

Arias A. La regresión de la hipertrofia ventricular izquierda mediante antihipertensivos reduce la muerte súbita. Evid actual pract ambul; 10(6); 171, Nov-Dic 2007. Wachtell K, Okin PM, Olsen MH, et al. Regression of electrocardiographic left ventricular hypertrophy during antihypertensive therapy and reduction in sudden cardiac death: the LIFE Study. Circulation 2007;116(7):700-5. PMID: 17664372.

\section{Bibliografia}

1. Lindholm LH; Ibsen H; Dahlof B; Devereux RB; et al. Cardiovascular morbidity and mortality in patients with diabetes in the Losartan Intervention For Endpoint reduction in hypertension study (LIFE): a randomized trial against atenolol. Lancet 2002;359:1004-10. Resumido en castellano en: http://www.fundacionmf.org.ar/hitalba-pagina-articulo.php?cod_producto=757 2. Prognostic Value of a New Electrocardiographic Method for Diagnosis of Left Ventricular Hypertrophy in Essential Hypertension. Verdecchia P, et al. J Am Coll Cardiol 1998, 3138390 . Disponible en castelano en: http://www. foroaps.org/hitalba-pagina-articulo.php?cod_producto=113

3. Devereux R, et al. Prognostic Significance ofLeft Ventricular Mass Change During Treatment of Hypertension. JAMA. 2004;292:2350-56

4. Levy D, et al. Determinants of Sensitivity and Specificity of Electrocardiographic Criteria for Left Ventricular Hypertrophy. Circulation 1990; 81:815-20.

5. Woythaler J, et al. Accuracy of Echocardiography Versus Electrocardiography in Detecting Left Ventricular Hypertrophy: Comparison With Postmortem Mass Measurements. Journal of American College of Cardiology 1983;2: 305-11

6. Devereux R, et al. Echocardiographic assesment of left ventricular hypertrophy: comparison to necropsy findings. Am J Cardiol 1986; 57:450-8.

7. Verdecchia P, et al. Prognostic Significance of Serial Changes in Left Ventricular Mass in Essential Hypertension. Circulation 1998; 97: 48-54

8. Guias de práctica clinica para el tratamiento de la hipertensión arterial 2007. Grupo de Trabajo para el Tratamiento de la Hipertensión Arterial de la Sociedad Europea de Hipertensión (ESH) y de la Sociedad Europea de Cardiologia (ESC). Mancia G, De Backer G, Dominiczak A, et al. Rev Esp Cardiol. 2007;60(9):968.e1-e94 